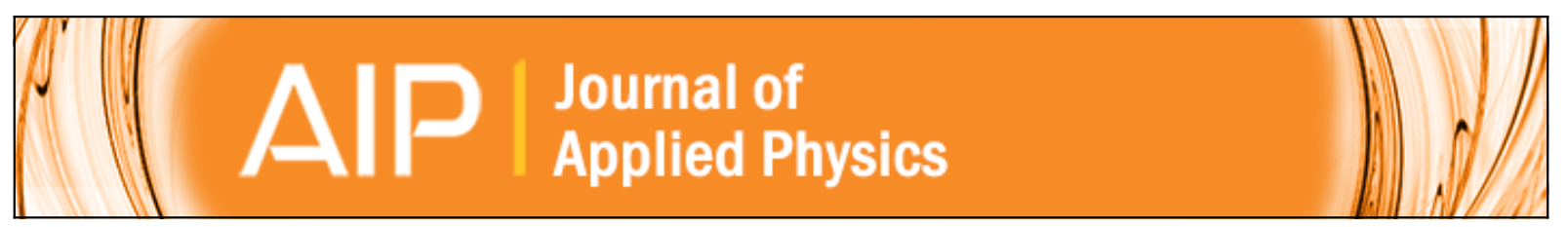

\title{
Wavelength-tunable infrared metamaterial by tailoring magnetic resonance condition with VO2 phase transition
}

Hao Wang, Yue Yang, and Liping Wang

Citation: Journal of Applied Physics 116, 123503 (2014); doi: 10.1063/1.4896525

View online: http://dx.doi.org/10.1063/1.4896525

View Table of Contents: http://scitation.aip.org/content/aip/journal/jap/116/12?ver=pdfcov

Published by the AIP Publishing

\section{Articles you may be interested in}

Electromagnetically induced transparency with large group index induced by simultaneously exciting the electric and the magnetic resonance

Appl. Phys. Lett. 105, 133514 (2014); 10.1063/1.4897194

Surface charge sensing by altering the phase transition in VO2

J. Appl. Phys. 116, 074511 (2014); 10.1063/1.4893577

Switchable wavelength-selective and diffuse metamaterial absorber/emitter with a phase transition spacer layer Appl. Phys. Lett. 105, 071907 (2014); 10.1063/1.4893616

Tunable waveguiding in electrically programmable $\mathrm{V} O 2$-based photonic crystals

J. Appl. Phys. 99, 113106 (2006); 10.1063/1.2200873

Phase transition-governed opal-VO2 photonic crystal

Appl. Phys. Lett. 79, 2127 (2001); 10.1063/1.1406144

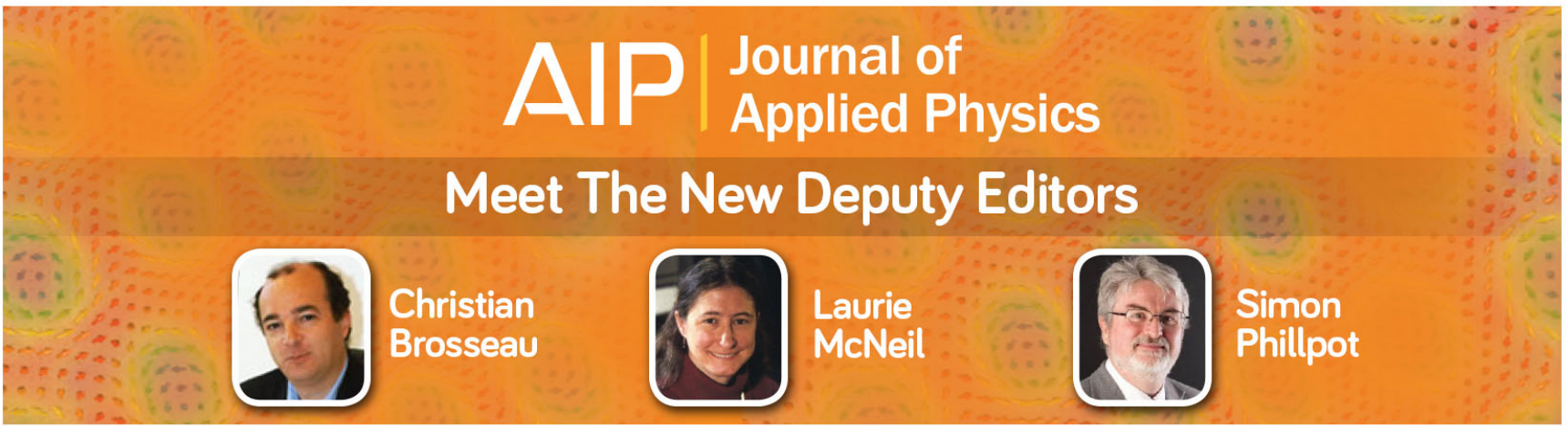




\title{
Wavelength-tunable infrared metamaterial by tailoring magnetic resonance condition with $\mathrm{VO}_{2}$ phase transition
}

\author{
Hao Wang, Yue Yang, and Liping Wang ${ }^{\text {a) }}$ \\ School for Engineering of Matter, Transport, and Energy, Arizona State University, \\ Tempe, Arizona 85287, USA
}

(Received 8 August 2014; accepted 15 September 2014; published online 23 September 2014)

\begin{abstract}
In this work, we report the design of a wavelength-tunable infrared metamaterial by tailoring magnetic resonance condition with the phase transition of vanadium dioxide $\left(\mathrm{VO}_{2}\right)$. Numerical simulation based on the finite-difference time-domain method shows a broad absorption peak at the wavelength of $10.9 \mu \mathrm{m}$ when $\mathrm{VO}_{2}$ is a metal, but it shifts to $15.1 \mu \mathrm{m}$ when $\mathrm{VO}_{2}$ changes to dielectric phase below its phase transition temperature of $68^{\circ} \mathrm{C}$. The large tunability of $38.5 \%$ in the resonance wavelength stems from the different excitation conditions of magnetic resonance mediated by plasmon in metallic $\mathrm{VO}_{2}$ but optical phonons in dielectric $\mathrm{VO}_{2}$. The physical mechanism is elucidated with the aid of electromagnetic field distribution at the resonance wavelengths. A hybrid magnetic resonance mode due to the plasmon-phonon coupling is also discussed. The results here would be beneficial for active control of thermal radiation in novel electronic, optical, and thermal devices. (C) 2014 AIP Publishing LLC. [http://dx.doi.org/10.1063/1.4896525]
\end{abstract}

\section{INTRODUCTION}

The unique phase transition behavior of vanadium dioxide $\left(\mathrm{VO}_{2}\right)^{1-3}$ has drawn lots of attentions recently and many applications have been found. Optical properties of $\mathrm{VO}_{2}$ change dramatically when the phase transition between dielectric and metal occurs at $68^{\circ} \mathrm{C}$, which can be thermally induced with temperature control. Applications of $\mathrm{VO}_{2}$ have been demonstrated in optical information storage, ${ }^{4}$ strain sensing, ${ }^{5}$ and lithium-ion batteries. ${ }^{6}$ Moreover, dielectric $\mathrm{VO}_{2}$ possesses several optical phonon modes in the infrared, which have been employed to modulate radiative heat trans$\mathrm{fer}^{7}$ in designing novel thermal devices such as vacuum thermal diodes/rectifiers ${ }^{8,9}$ and thermal transistors. ${ }^{10}$

Progresses have been made recently in designing tunable metamaterials made of phase transition $\mathrm{VO}_{2}$. Dicken et al. demonstrated a frequency-tunable metamaterial by depositing split-ring resonators on a $\mathrm{VO}_{2}$ film. ${ }^{11}$ Kats et al. showed $\sim 10 \%$ resonance wavelength tunability with a plasmonic antenna array on a $\mathrm{VO}_{2}$ film in the mid-infrared. ${ }^{12}$ They also demonstrated an ultra-thin tunable perfect absorber based on the $\mathrm{VO}_{2}$ phase transition. ${ }^{13}$ More recently, a switchable thermal antenna with periodically patterned $\mathrm{VO}_{2}$ has been proposed. ${ }^{14}$ Designs reported in Refs. 12 and 14 were realized by modulating the excitation condition of surface plasmon polariton (SPP) at the interface between subwavelength plasmonic nanostructures and the supporting $\mathrm{VO}_{2}$ film upon phase transition.

Magnetic resonance has been studied intensively for designing selective thermal emitters ${ }^{15,16}$ and perfect metamaterial absorbers. ${ }^{17}$ Magnetic resonance occurs when external electromagnetic wave couples with magnetic resonance excited inside the metamaterial structures typically in a metal-insulator-metal configuration, resulting in strong

\footnotetext{
a) Author to whom correspondence should be addressed. Electronic mail: liping.wang@asu.edu
}

absorption or emission at the selected resonance frequency. Note that, magnetic resonance can be also excited in the mid-infrared regime with polar materials, mediated by optical phonons, rather than plasmon in metals. An infrared selective emitter made of $\mathrm{SiC}$ has been demonstrated by exciting magnetic resonance within its phonon absorption band. ${ }^{18}$ The electrical current induced by the resonant magnetic field is realized by the high-frequency vibration of ions in $\mathrm{SiC}$, rather than free charges in metals.

In this study, we present a tunable infrared metamaterial by exciting magnetic resonance at different conditions with either metallic or dielectric $\mathrm{VO}_{2}$, leading to highly tunable resonant wavelength upon the phase transition of $\mathrm{VO}_{2}$. Figure 1 depicts the proposed tunable metamaterial structure, which is made of a one-dimensional $\mathrm{VO}_{2}$ periodic grating structure (period $\Lambda=1.5 \mu \mathrm{m}$ and strip width $w=1.25 \mu \mathrm{m}$ ) on stacked $\mathrm{MgF}_{2}$ and $\mathrm{VO}_{2}$ layers. The thicknesses of the $\mathrm{VO}_{2}$ grating and thin films are $h=0.5 \mu \mathrm{m}, d_{1}=0.3 \mu \mathrm{m}$, and $d_{2}=1 \mu \mathrm{m}$, respectively. In practice, the $\mathrm{VO}_{2}$ grating could be formed by the

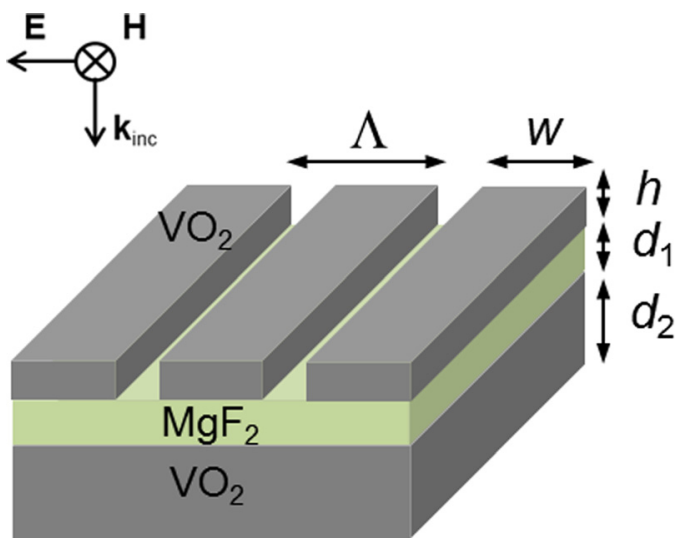

FIG. 1. Proposed 1D tunable structure with period $\Lambda=1.5 \mu \mathrm{m}$, strip width $w=1.25 \mu \mathrm{m}$, layer thicknesses $h=0.5 \mu \mathrm{m}, d_{1}=0.3 \mu \mathrm{m}$, and $d_{2}=1 \mu \mathrm{m}$. The phase transition of $\mathrm{VO}_{2}$ can be controlled by modulating the temperature. 
strain mismatch method from a $\mathrm{VO}_{2}$ film on a flexible substrate, ${ }^{19,20}$ while the temperature of the structure can be modulated to thermally control $\mathrm{VO}_{2}$ phase transition.

\section{NUMERICAL METHODS}

When the temperature is above $68^{\circ} \mathrm{C}, \mathrm{VO}_{2}$ is an isotropic metal, whose electrical permittivity $\varepsilon_{\mathrm{m}}$ can be described by a Drude model as ${ }^{1}$

$$
\varepsilon_{\mathrm{m}}=-\varepsilon_{\infty} \frac{\omega_{p}^{2}}{\omega^{2}+i \omega \Gamma}
$$

where $\omega$ is angular frequency, $\varepsilon_{\infty}=9$ is the high-frequency constant, $\omega_{\mathrm{p}}=8000 \mathrm{~cm}^{-1}$ is the plasma frequency, and $\Gamma=10000 \mathrm{~cm}^{-1}$ is the collision frequency. When the temperature is below $68^{\circ} \mathrm{C}, \mathrm{VO}_{2}$ becomes dielectric but with uniaxial anisotropy. Considering (200)-oriented crystal $\mathrm{VO}_{2}$ with optical axis normal to the surface, ${ }^{1}$ it exhibits ordinary dielectric response denoted as $\varepsilon_{O}$ when incident electric field is perpendicular to optical axis, and extraordinary response $\varepsilon_{\mathrm{E}}$ when electric field is parallel to optical axis. Both components can be described by a classical oscillator model as

$$
\varepsilon(\omega)=\varepsilon_{\infty}+\sum_{j=1}^{N} \frac{S_{j} \omega_{j}^{2}}{\omega_{j}^{2}-i \gamma_{j} \omega-\omega^{2}},
$$

where $\omega_{j}$ is the phonon vibration frequency, $\gamma_{j}$ is the scattering rate, $S_{j}$ represents the oscillation strength, and $j$ is the phonon mode index. The values for each parameter can be found from Ref. 1 for both ordinary $\left(\varepsilon_{\mathrm{O}}\right)$ and extraordinary $\left(\varepsilon_{\mathrm{E}}\right)$ components with a total of eight phonon modes for $\varepsilon_{\mathrm{O}}$ and nine modes for $\varepsilon_{\mathrm{E}}$. In the simulation, the permittivity tensor was employed to consider the uniaxial anisotropy of dielectric $\mathrm{VO}_{2}$,

$$
\overline{\bar{\varepsilon}}_{\text {dielectric }}=\left(\begin{array}{ccc}
\varepsilon_{\mathrm{O}} & 0 & 0 \\
0 & \varepsilon_{\mathrm{O}} & 0 \\
0 & 0 & \varepsilon_{\mathrm{E}}
\end{array}\right) .
$$

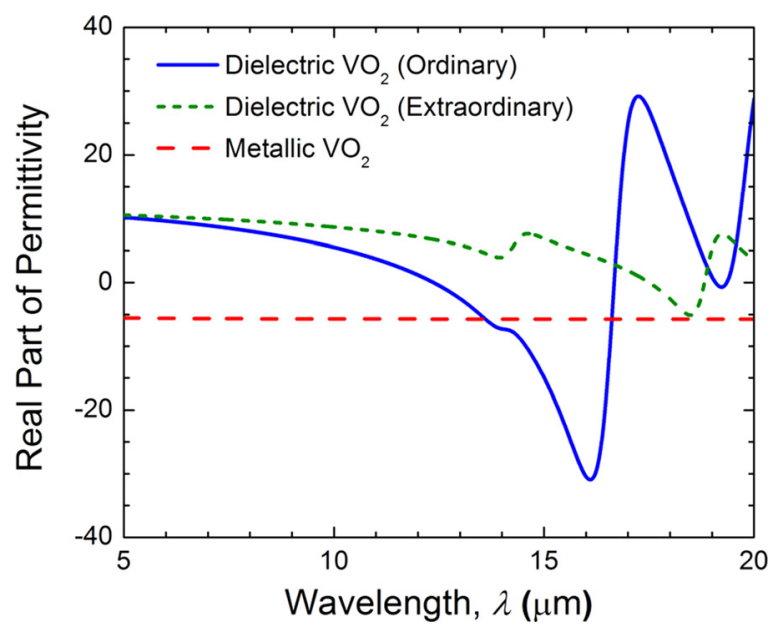

FIG. 2. Real parts of permittivity of $\mathrm{VO}_{2}$ at either metallic or dielectric phase, which exhibits both ordinary and extraordinary dielectric response.
Figure 2 plots the real parts for the permittivity of metallic and dielectric $\mathrm{VO}_{2}$ in the mid-infrared regime from $5 \mu \mathrm{m}$ to $20 \mu \mathrm{m}$ in wavelength. The metallic phase exhibits a negative real part of permittivity, which is crucial to excite plasmonic resonances as found in most noble metals. ${ }^{21}$ On the other hand, there exist several phonon modes in both ordinary and extraordinary components of the dielectric phase. As a result, negative real part of permittivity exists in the wavelengths from $12.4 \mu \mathrm{m}$ to $16.7 \mu \mathrm{m}$ for $\varepsilon_{\mathrm{O}}$ and from $17.5 \mu \mathrm{m}$ to $18.8 \mu \mathrm{m}$ for $\varepsilon_{\mathrm{E}}$, respectively. As pointed out by Ref. 18, negative permittivity is required to excite phononmediated magnetic resonance in polar materials. The unique material properties of metallic and dielectric $\mathrm{VO}_{2}$ suggest the potential in exciting magnetic resonance at both phases.

The finite-difference time-domain method (Lumerical Solutions, Inc.) was used to numerically calculate the spectral reflectance $R$ and transmittance $T$ of the proposed tunable metamaterial above and below the $\mathrm{VO}_{2}$ phase transition temperature of $68^{\circ} \mathrm{C}$. The optical constants of $\mathrm{MgF}_{2}$ were obtained from Palik's data. ${ }^{22}$ A linearly polarized plane wave was incident normally onto the metamaterial structure with transverse-magnetic (TM) incidence, in which magnetic field is along the grating groove direction. Note that, magnetic resonance can be excited only at TM polarization in 1D grating based metamaterials. ${ }^{15,16}$ A numerical error less than $2 \%$ was verified with sufficiently fine mesh sizes. The spectral normal absorptance in the infrared region was thus obtained by $\alpha=1-R-T$ based on energy balance.

\section{RESULTS AND DISCUSSIONS}

\section{A. Tunable resonance absorption with $\mathrm{VO}_{2}$ phase transition}

As shown in Fig. 3, when the temperature is above $68^{\circ} \mathrm{C}$, the $\mathrm{VO}_{2}$ is at metallic phase and the metamaterial exhibits a broad absorption band peaked at the wavelength of $10.9 \mu \mathrm{m}$ with almost $100 \%$ absorption. However, when $\mathrm{VO}_{2}$ becomes dielectric at temperatures less than $68^{\circ} \mathrm{C}$, the absorption band is narrower and shifts to the peak

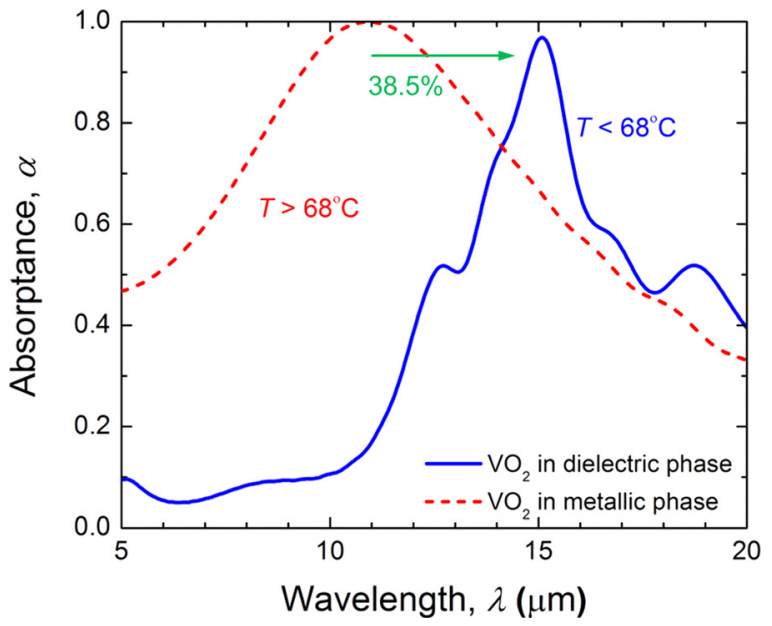

FIG. 3. Simulated normal absorptance of proposed tunable absorber in the mid-infrared upon $\mathrm{VO}_{2}$ phase transition, showing a relative $38.5 \%$ shift of resonant absorption peak wavelength. 
wavelength of $15.1 \mu \mathrm{m}$ with maximum absorptance of 0.97 , resulting in a relative $38.5 \%$ peak wavelength shift upon the $\mathrm{VO}_{2}$ phase transition from metal to dielectric induced thermally. Note that there exist three bumps on the shoulder of absorption peak around $13 \mu \mathrm{m}, 16.5 \mu \mathrm{m}$, and $19 \mu \mathrm{m}$, which are caused by the abrupt change in the optical properties of dielectric $\mathrm{VO}_{2}$ associated with several phonon absorption modes at these wavelengths.

In fact, both absorption peaks are caused by the excitations of magnetic resonance at both phases of $\mathrm{VO}_{2}$. But the fundamental difference is that, one is assisted by free charges or plasmon in metallic phase, while the other is mediated by optical phonons in its dielectric phase. The different resonance conditions and thereby the resulting large resonance wavelength shift are due to different optical behaviors of different energy carriers that excite the magnetic resonances.

\section{B. Electromagnetic field distribution at magnetic resonance}

To illustrate the underlying mechanism responsible for the large absorption peaks, electromagnetic field distributions at the cross section of the metamaterial structure were plotted at the resonance wavelengths with metallic and dielectric phases of $\mathrm{VO}_{2}$, as shown in Figs. 4(a) and 4(b), respectively. The arrows indicate the strength and direction of the electric field vectors, while the contour shows magnetic field strength normalized to the incidence as $\left|H / H_{0}\right|^{2}$ at different locations.
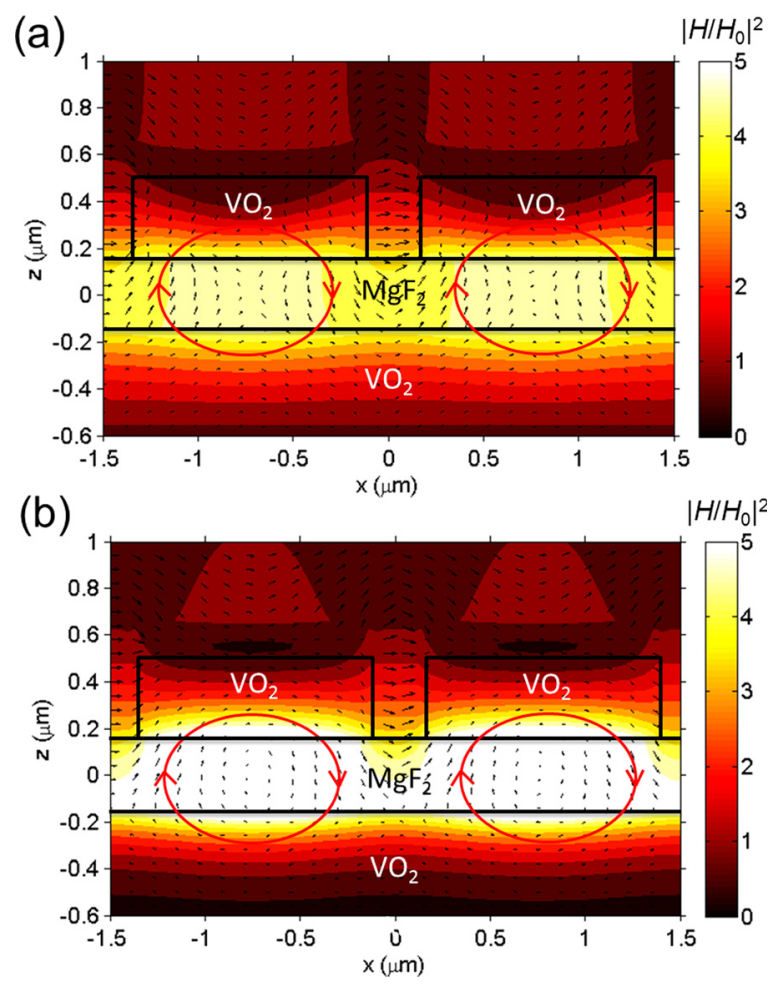

FIG. 4. Electromagnetic field distribution at resonance peak wavelengths when $\mathrm{VO}_{2}$ is at (a) metallic or (b) dielectric phase. The field patterns show the exact behavior of magnetic resonance with both phases of $\mathrm{VO}_{2}$, but assisted by plasmon in metallic $\mathrm{VO}_{2}$ and mediated by optical phonons in dielectric $\mathrm{VO}_{2}$, respectively.
When $\mathrm{VO}_{2}$ is at metallic phase, the electric field vectors inside the $\mathrm{MgF}_{2}$ layer underneath the $\mathrm{VO}_{2}$ strips indicate an anti-parallel current loop, along with the strong localization of magnetic field, as shown in Fig. 4(a). The localized energy is more than five times higher than the incidence. This is the exact behavior of magnetic resonance that has been intensively studied in similar 1D grating based metamaterials. ${ }^{15,16}$ Due to the oscillating movement of free charges in metallic $\mathrm{VO}_{2}$, the sandwiched $\mathrm{MgF}_{2}$ layer serves as a capacitor, while top metallic $\mathrm{VO}_{2}$ strip and the bottom metallic $\mathrm{VO}_{2}$ film function as inductors, forming a resonant alternating-current circuit. When the magnetic resonance occurs, the external electromagnetic energy at the resonant wavelength of 10.9 $\mu \mathrm{m}$ is coupled with the oscillating plasmon, resulting in almost $100 \%$ absorption inside the metamaterial structure.

When $\mathrm{VO}_{2}$ becomes dielectric with the temperature below $68^{\circ} \mathrm{C}$, the electromagnetic field shown in Fig. 4(b) presents a similar behavior of magnetic resonance with an induced anti-parallel electric current loop and confined magnetic field inside the $\mathrm{MgF}_{2}$ layer but at a different resonance wavelength of $15.1 \mu \mathrm{m}$. The localized energy strength is about five times to the incidence. Note that, this resonant wavelength is within the phonon absorption band of the ordinary component of dielectric $\mathrm{VO}_{2}$, in which negative permittivity exists. When optical phonons vibrate at high frequency, the fast movements of bound charges or ions form oscillating electric currents and an inductor-capacitor resonant circuit, resulting in the excitation of magnetic resonance. Since the energy carrier changes from free electrons to optical phonons upon the phase transition of $\mathrm{VO}_{2}$ from metal to dielectric, a large shift in resonance wavelengths occurs. It should be noted that, similar to the surface phonon polariton with polar materials, ${ }^{23}$ which is a counterpart of SPP in the infrared regime, phonon-mediated magnetic resonance $^{18}$ is the counterpart of magnetic resonance in plasmonic metamaterials made of metallic nanostructures. ${ }^{15-17}$

\section{Hybrid magnetic resonance due to the phonon-plasmon coupling}

Finally, we would like to show that a hybrid magnetic resonance mode could also occur by the phonon-plasmon coupling from a modified tunable metamaterial by replacing the bottom $\mathrm{VO}_{2}$ layer with a gold film, as shown in the inset of Fig. 5. The period and strip width of the top $\mathrm{VO}_{2}$ grating are kept unchanged, while the thicknesses of the grating and the $\mathrm{MgF}_{2}$ spacer layer are $h=d_{1}=0.5 \mu \mathrm{m}$.

The spectral normal absorptance of the hybrid structure at TM waves is plotted in Fig. 5. When $\mathrm{VO}_{2}$ is in either metallic or dielectric phase, the absorption peaks remain almost at the same resonance wavelengths, suggesting that magnetic resonance can still be excited in both phases of $\mathrm{VO}_{2}$. However, maximum absorptance drops slightly to 0.85 for the peak with metallic $\mathrm{VO}_{2}$, while the absorption peak with dielectric $\mathrm{VO}_{2}$ becomes narrower, after the bottom $\mathrm{VO}_{2}$ film was replaced by a gold substrate. The successful excitation of magnetic resonance between metallic $\mathrm{VO}_{2}$ strips and the bottom gold film is easy to understand, as there exist free charges in both metals. The peak absorptance drops due to 


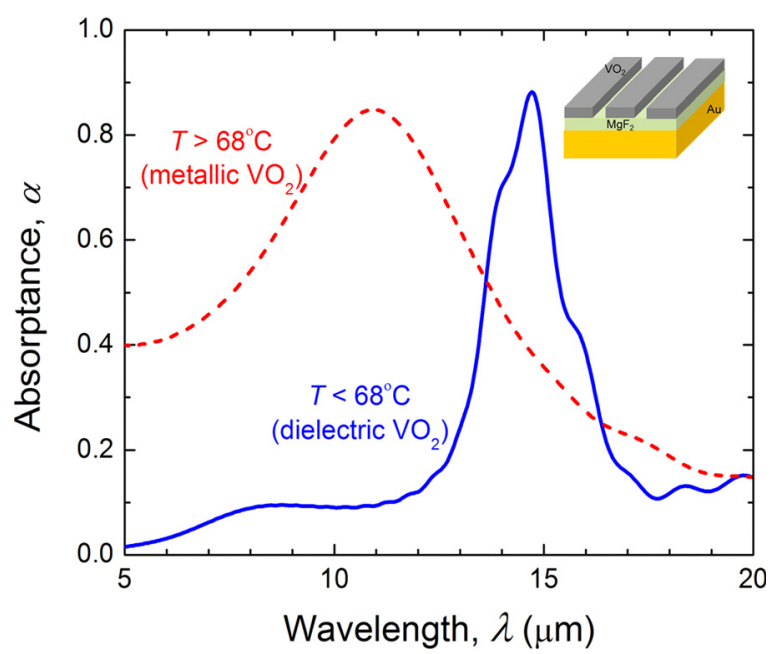

FIG. 5. Normal absorptance of the tunable structure with gold substrate instead of the bottom $\mathrm{VO}_{2}$ layer. The absorption peaks still exist because of a hybrid magnetic resonance mode due to phonon-plasmon coupling between top dielectric $\mathrm{VO}_{2}$ and bottom gold.

the plasmonic coupling between metallic $\mathrm{VO}_{2}$ and $\mathrm{Au}$, whose strength is weaker compared to that between two identical materials with matching plasmonic properties. On the other hand, it would be expected that it would fail to excite phonon-mediated magnetic resonance due to the removal of the bottom $\mathrm{VO}_{2}$ film. Surprisingly, the strong absorption with dielectric $\mathrm{VO}_{2}$ could still occur. This can be understood by the excitation of a hybrid magnetic resonance mode due to the strong coupling between optical phonons in dielectric $\mathrm{VO}_{2}$ and plasmon in the bottom gold substrate. The high-frequency vibration of optical phonons at the top interface of the $\mathrm{MgF}_{2}$ spacer along with the movement of plasmon at the bottom interface could still form a close-loop inductor-capacitor circuit, which successfully excites magnetic resonance at the wavelength of $14.8 \mu \mathrm{m}$. Note that the absorption peak becomes narrower because $\mathrm{Au}$ has less intrinsic loss compared with the $\mathrm{VO}_{2}$ substrate.

\section{CONCLUSIONS}

In summary, we have numerically demonstrated a wavelength-tunable metamaterial by tailoring magnetic resonance conditions with phase transition of $\mathrm{VO}_{2}$. The absorption peak shifts from $10.9 \mu \mathrm{m}$ to $15.1 \mu \mathrm{m}$ upon the $\mathrm{VO}_{2}$ phase transition from metal to dielectric, resulting in a relative $38.5 \%$ shift in the peak wavelength. The underlying physical mechanisms lie in the plasmon-assisted magnetic resonance in metallic $\mathrm{VO}_{2}$ and phonon-mediated counterpart in dielectric $\mathrm{VO}_{2}$, which leads to different resonance wavelengths. A hybrid magnetic resonance mode due to phonon-plasmon coupling was also discussed when replacing the bottom $\mathrm{VO}_{2}$ layer with a gold film, which could simplify the metamaterial design in practice. The wavelength-tunable metamaterial absorber or emitter could find applications in tunable infrared detectors and coherent thermal emitters. The insights and understanding gained in this work will facilitate the design of novel tunable metamaterials for active control of thermal radiation in electronic, optical, and thermal devices.

\section{ACKNOWLEDGMENTS}

This work was supported by the New Faculty Startup fund at Arizona State University.

${ }^{1}$ A. Barker, Jr., H. Verleur, and H. Guggenheim, Phys. Rev. Lett. 17, 1286 (1966).

${ }^{2}$ M. M. Qazilbash, M. Brehm, B.-G. Chae, P.-C. Ho, G. O. Andreev, B.-J. Kim, S. J. Yun, A. V. Balatsky, M. B. Maple, F. Keilmann, H.-T. Kim, and D. N. Basov, Science 318, 1750 (2007).

${ }^{3}$ M. M. Qazilbash, M. Brehm, G. O. Andreev, A. Frenzel, P.-C. Ho, B.-G. Chae, B.-J. Kim, S. J. Yun, H.-T. Kim, A. V. Balatsky, O. G. Shpyrko, M. B. Maple, F. Keilmann, and D. N. Basov, Phys. Rev. B 79, 075107 (2009). ${ }^{4}$ I. Balberg and S. Trokman, J. Appl. Phys. 46, 2111 (1975).

${ }^{5}$ B. Hu, Y. Ding, W. Chen, D. Kulkarni, Y. Shen, V. V. Tsukruk, and Z. L. Wang, Adv. Mater. 22, 5134 (2010).

${ }^{6}$ H. Liu, Y. Wang, K. Wang, E. Hosono, and H. Zhou, J. Mater. Chem. 19, 2835 (2009).

${ }^{7}$ P. van Zwol, K. Joulain, P. Ben-Abdallah, and J. Chevrier, Phys. Rev. B 84, 161413 (2011).

${ }^{8}$ Y. Yang, S. Basu, and L. P. Wang, Appl. Phys. Lett. 103, 163101 (2013).

${ }^{9}$ P. Ben-Abdallah and S.-A. Biehs, Appl. Phys. Lett. 103, 191907 (2013).

${ }^{10}$ P. Ben-Abdallah and S.-A. Biehs, Phys. Rev. Lett. 112, 044301 (2014).

${ }^{11}$ M. J. Dicken, K. Aydin, I. M. Pryce, L. A. Sweatlock, E. M. Boyd, S. Walavalkar, J. Ma, and H. A. Atwater, Opt. Express 17, 18330 (2009).

${ }^{12}$ M. A. Kats, R. Blanchard, P. Genevet, Z. Yang, M. M. Qazilbash, D. Basov, S. Ramanathan, and F. Capasso, Opt. Lett. 38, 368 (2013).

${ }^{13}$ M. A. Kats, D. Sharma, J. Lin, P. Genevet, R. Blanchard, Z. Yang, M. M. Qazilbash, D. Basov, S. Ramanathan, and F. Capasso, Appl. Phys. Lett. 101, 221101 (2012).

${ }^{14}$ P. Ben-Abdallah, P. Benisty, H. Benisty, and M. Besbes, J. Appl. Phys. 116, 034306 (2014).

${ }^{15}$ B.-J. Lee, L. P. Wang, and Z. M. Zhang, Opt. Express 16, 11328 (2008).

${ }^{16}$ L. P. Wang and Z. M. Zhang, Appl. Phys. Lett. 100, 063902 (2012).

${ }^{17}$ H. Wang and L. P. Wang, Opt. Express 21, A1078 (2013).

${ }^{18}$ L. P. Wang and Z. M. Zhang, Opt. Express 19, A126 (2011).

${ }^{19}$ M. Liu, M. Wagner, J. Zhang, A. McLeod, S. Kittiwatanakul, Z. Fei, E. Abreu, M. Goldflam, A. J. Sternbach, and S. Dai, Appl. Phys. Lett. 104, 121905 (2014).

${ }^{20}$ M. Liu, M. Wagner, E. Abreu, S. Kittiwatanakul, A. McLeod, Z. Fei, M. Goldflam, S. Dai, M. Fogler, and J. Lu, Phys. Rev. Lett. 111, 096602 (2013).

${ }^{21}$ S. A. Maier, Plasmonics: Fundamentals and Applications (Springer, 2007).

${ }^{22}$ E. D. Palik, Handbook of Optical Constants of Solids (Academic, 1985).

${ }^{23}$ J.-J. Greffet, R. Carminati, K. Joulain, J.-P. Mulet, S. Mainguy, and Y. Chen, Nature 416, 61 (2002). 